Macedonian Pharmaceutical Bulletin, 66 (Suppl 1) 217 - 218 (2020)

Online ISSN 1857 - 8969

UDC: $616.248-085.458$

DOI: 10.33320/maced.pharm.bull.2020.66.03.108

Short communication

\title{
Leukotriene receptor antagonist (LTRA) added to regular preventive therapy: inhaled corticosteroids and long- acting beta agonists (ICS/LABA) in patients with severe uncontrolled asthma
}

\author{
Elena Jovanovska-Janeva ${ }^{1}$, Dejan Dokic ${ }^{1}$, Biserka Kaeva ${ }^{1}$, Gorica Breskovska ${ }^{1}$, \\ Zlatica Goseva ${ }^{1}$, Zoran Arsovski ${ }^{1}$, Olivera Krstic Nakovska ${ }^{1}$, Dejan Trajkov ${ }^{2}$, \\ Magdalena Dimitrova Genadieva ${ }^{3}$ \\ ${ }^{1}$ PHI University Clinic of Pulmonology and Allergy, Majka Tereza 47, 1000 Skopje, North Macedonia \\ ${ }^{2}$ Institute of Immunobiology and Human Genetics, Majka Tereza 47, 1000 Skopje, North Macedonia \\ ${ }^{3}$ PHI University Clinic of Gastroenterohepatology, Majka Tereza 47, 1000 Skopje, North Macedonia
}

\section{Introduction}

Asthma is a worldwide problem and also is one of the most common chronic diseases worldwide 334 million patients suffered from asthma. It is estimated that number is expected to reach 400 million by 2025 . Prevalence was increasing in many countries, especially in children $-6 \%$ and in adult $10 \%$. There are 250,000 deaths annually (GINA, 2019). In the Republic of North Macedonia 100,000 or $5 \%$ of the population suffers from asthma (Cvetanov, 2006).

Asthma is a heterogeneous disease, characterized by chronic airway inflammation of the airways in which many cells play a role, in particular mast cells, eosinophils, and $\mathrm{T}$ lymphocytes. It is defined by the history of respiratory symptoms such as wheeze, shortness of breath, chest tightness and cough that vary over time and in intensity, together with variable expiratory airflow limitation that is at least partly reversible either spontaneously or with treatment (GINA, 2019).

In this chronic inflammation are involve more than 100 mediators and the interleukins take central place in this inflammation. The interleukins are cytokines that stimulate the proliferation and differentiation of immune cells. T cells play a key role in coordinating the immune response in asthma. Th2 cells produce, IL-4, IL-5, IL-9, IL-13, GM-CSF and IL-25, IL-31, IL-33 that are responsible for chronic eosinophilic inflammation, inflammation in allergic diseases, including and asthma (Hamid et al., 2003; Zhu et al., 2010)

The aim of this study was to determine the effect of combined therapy: leukotriene receptor antagonist (LTRA) added to regular preventive therapy consisted of inhaled corticosteroids and long-acting beta agonists (ICS/LABA) in patients with severe uncontrolled asthma, by analyzing of IL-5, forced expiratory volume in the first second (FEV1) and Symptom score at the beginning and after 6 months of therapy.

\section{Materials and Methods}

The study included 29 patients with severe uncontrolled asthma. They were treated with LTRA, Montelucast $10 \mathrm{mg} /$ daily and combined therapy of (ICS/LABA) 500/50 mcg twice daily in duration of 6 months. In each of them were measured serum IL-

*eli.janeva@yahoo.com 
5 levels by the ELISA method before the treatment and after 6 months of therapy at the Institute of Immunobiology and Human Genetics, Faculty of Medicine, Skopje. Spirometry was done at the Clinic of Pulmonology and Allergy, Skopje. We determined FEV1 and assessed Symptom score with 5-point Likert scale of breathlessness at the beginning and after 6 months of therapy.

Inclusion Criteria included uncontrolled severe persistent asthma patients. The classification was according to the actual version of the GINA guidelines (GINA, 2019) and Guidelines of National Asthma Education Prevention Program (NAEPP, 2007). The age of the patients was 18-70 years. Exclusion criteria included: pregnancy, severe diseases of the immune, endocrine, hematological cardiac, renal, gastrointestinal and neurological system, psychiatric disorders and neoplastic diseases.

Results were statistically elaborated according to the Wilcoxon Pairs Test and T-test for Dependent Samples. The significances values were taken $\mathrm{p}<0.05$ and a highly significant $\mathrm{p}<0.01$.

\section{Results and Discussion}

The obtained results of IL-5 showed that the level of IL-5 before the start of therapy were much higher and that treatment significantly reduced their value $(Z=4.64 ; p=0.000004)$. The difference in the average value of FEV1 before and after therapy was statistically significant $(\mathrm{t}=7.56 ; \mathrm{p}=0.000000)$. There was a difference in Symptom score before and after 6 month therapy $(\mathrm{Z}=4.54 ; \mathrm{p}=0.000006)$, pointing to significant improvement in asthma symptoms, because the patients after treatment were pleaded with much lower Symptom score.

The mechanism of action of the LTRA is based on counteracting the effects of cysLTs at their receptor site (CysLT1-receptor) within the airways. This results in a dual effect: suppression of the airway inflammation and mild bronchodilator properties. When is added to ICS/LABA the act complementary with improving symptoms, lung function, significant reduction in exacerbations and inflammation parameters. (Diamant and Van der Molen, 2005). Montelukast may affect eosinophilic reactions by reducing IL-5 synthesis and circulating in the asthmatic airways, and consequently to reduce airway inflammation and blood eosinophils in asthma (Hamid et al., 2003). In a smaller, open label study of 313 patients with insufficiently controlled patients on combination therapy (ICS/LABA) they observed improvement in asthma symptoms and pulmonary function with add-on LTRA therapy after 2 months of therapy (Dupont et al., 2005).

\section{Conclusion}

Combination therapy with ICS/LABA represents the gold standard in the treatment of severe uncontrolled asthma and it is safe and effective treatment. However, not all patients have well control of asthma. In such patients, there is a need for additional add-on therapy, such as treatment with LTRA. Combination ICS/LABA improve symptoms and lung function, and the addition of LTRA offers additional suppression of airway inflammation, as confirmed in our study. Future studies should confirm this option.

\section{References}

Cvetanov, V., 2006. Allergic Diseases in R. Macedonia, first ed. ITM and MZBKIA, Skopje. Bronchial asthma, 151-174.

Diamant, Z., Van der Molen, T., 2005. Treating asthma: is there place for leukotriene receptor antagonists? Respiratory Medicine 99, 655-662.

Dupont, L., Potvin, E., Korn, D., Lachman, A., Dramaix, M., Gusman, J., Peche, R., 2005. Improving asthma control in patients suboptimally controlled on inhaled steroids and long acting beta2 - agonists: Addition of Montelukast in an open-label pilot study. Curr. Med. Res. Opin. 21(6), $863-869$

Global Initiative for Asthma (GINA), 2019. GINA Report 2019, Global Strategy for Asthma Management and Prevention. Available at: http://www.ginasthma.org.

Hamid, Q., Tulic, K.M., Liu, C.M., Moqbel, R., 2003. Inflammatory cells in asthma: Mechanisms and implications for therapy. J. Allergy Clin. Immunol. 111, S5-17.

National Asthma Education and Prevention Program (NAEPP) 2007, Expert Panel Report 3. National Heart, Lung and Blood Institute. Guidelines for the Diagnosis and Management of Asthma. Accessed: March 13, 2020.

Zhu, J., Yamane, H., Paul, W.E., 2010. Differentiation of effectors' CD4 T cell populations. Ann. Rev. Immunol. 28, 445-489. 